\title{
ON THE CRITICAL GALTON-WATSON PROCESS WITH IMMIGRATION
}

\author{
A. G. PAKES \\ Monash University \\ (Received 4th September 1969) \\ Communicated by P. D. Finch
}

\section{Introduction}

Consider a Galton-Watson process in which each individual reproduces independently of all others and has probability $a_{j}(j=0,1, \cdots)$ of giving rise to $j$ progeny in the following generation and in which there is an independent immigration component where $b_{j}(j=0,1, \cdots)$ is the probability that $j$ individuals enter the population at each generation. Then letting $X_{n}(n=0,1, \cdots)$ be the population size of the $n$-th generation, it is known (Heathcote [4], [5]) that $\left\{X_{n}\right\}$ defines a Markov chain on the non-negative integers. Unless otherwise stated, we shall consider only those offspring and immigration distributions that make the Markov chain $\left\{X_{n}\right\}$ irreducible and aperiodic.

Heathcote [5] has shown that in the case $\alpha=\sum_{j} j a_{j}<1$, a necessary and sufficient condition for $\left\{X_{n}\right\}$ to be positive-recurrent is that $\sum_{j=1}^{\infty} b_{j} \log j<\infty$. Seneta [7] has shown that if $\alpha=1$ and if $2 \gamma=\sum_{j} j(j-1) a_{j}=\infty$ then it is possible for $\left\{X_{n}\right\}$ to be positive-recurrent.

In this paper we consider the case $\alpha=1$ and $\beta, \gamma<\infty$ where $\beta=\sum_{j} j b_{j}$ is the mean of the immigration distribution. We shall show that the Markov chain $\left\{X_{n}\right\}$ may be either null-recurrent or transient. In the case of null-recurrence we obtain some information on the occupation times of the zero state. Finally, in the last section we show that $X_{n} / n$ tends in distribution to a random variable having a gamma distribution.

\section{Classification of the Markov chain $\left(X_{n}\right)$}

Let $p_{i j}^{(n)}(i, j, n=0,1, \cdots)$ be the $n$-step transition probability from state $i$ to $j$ and let $P_{i}^{(n)}(x)=\sum_{j=0}^{\infty} p_{i j}^{(n)} x^{j} \quad(|x|<1)$. Then letting $A(x)=\sum_{j=0}^{\infty} a_{j} x^{j}$ and $B(x)=\sum_{j=0}^{\infty} b_{j} x^{j}(|x|<1)$ be the probability generating functions of the offspring and immigration distributions respectively, it is not difficult to show that

$$
P_{i}^{(n)}(x)=\left[A_{n}(x)\right]_{m=0}^{i-1} \prod_{m} B\left[A_{m}(x)\right]
$$


where $A_{0}(x)=x$ and $A_{n+1}(x)=A\left(A_{n}(x)\right)(n=0,1, \cdots)$, so that in particular we have

$$
p_{00}^{(n)}=B(0) \prod_{m=1}^{n-1} B\left(A_{m}(0)\right)
$$

and it is clear that $p_{00}^{(n)}$ is a non increasing sequence.

Before stating theorem 1, we shall state a theorem which will play a key role in our work.

TheORem A. (Kesten, Ney and Spitzer [6]). If $\alpha=1$ and $0<\gamma<\infty$ and

$$
1 /(1-x)+n \gamma-1 /\left[1-A_{n}(x)\right]=h_{n}(x) \quad(0 \leqq x<1)
$$

then $\lim _{n \rightarrow \infty} h_{n}(x) / n=0$ uniformly in $0 \leqq x<1$. Furthermore, $\quad h_{n}(x)=$ $\sum_{m=0}^{n-1} \delta\left(A_{m}(x)\right)$ where $\delta(x)$ satisfies the inequality

$$
\frac{-\gamma^{2}(1-x)}{1-a_{0}} \leqq \delta(x) \leqq \varepsilon(x) \quad(0 \leqq x<1)
$$

where $0 \leqq \varepsilon(x)=\gamma-[A(x)-x] /(1-x)^{2} \leqq \gamma$ and $\varepsilon(x)$ is non-increasing in $x$ and $\varepsilon(x) \downarrow 0(x \uparrow 1)$.

Observe that our assumption of irreducibility implies that $\gamma>0$.

THEOREM 1. Let $\alpha=1$ and $\beta, \gamma<\infty$, then the Markov chain $\left\{X_{n}\right\}$ is not positive-recurrent. Further, let $\sigma=\beta / \gamma$, then $\left\{X_{n}\right\}$ is null-recurrent if $\sigma<1$ and transient if $\sigma>1$. Define $\varepsilon(x)$ as in theorem $A$ and let $\varepsilon(x)=O\left[(1-x)^{\delta}\right](x \uparrow 1)$ for some $\delta>0$. If $B^{\prime \prime}(1-)<\infty$ then $p_{00}^{(n)} \sim C n^{-\sigma}$ as $n \rightarrow \infty$ where $C$ is a finite, positive constant, and in particular, if $\sigma=1$ then $\left\{X_{n}\right\}$ is null-recurrent.

Proof. By irreducibility and aperiodicity, the Markov chain $\left\{X_{n}\right\}$ is not positive-recurrent if $\lim _{n \rightarrow \infty} p_{00}^{(n)}=0$, that is if the infinite product $\prod_{m=1}^{\infty} B\left(A_{m}(0)\right)$ diverges to zero. (Observe that irreducibility implies that $B(0)>0$.) It is known (Seneta [7]) that if $\beta<\infty$ then a necessary and sufficient condition for this is the divergence of the integral $\int_{0}^{1}[(1-x) /(A(x)-x)] d x$. By Taylor's theorem, $A(x)$ $=1-(1-x)+(1-x)^{2} A^{\prime \prime}(\theta) / 2(x<\theta<1)$ and so if $0<A^{\prime \prime}(1-)<\infty$, we see that the integrand is bounded below by $[(1-x) \gamma]^{-1}$ and so the integral diverges.

Thus the Markov chain will be transient or null-recurrent according as the series $\sum_{n=0}^{\infty} p_{00}^{(n)}$ converges or diverges, and by Raabe's test (Ferrar [2]) the first alternative will occur if $\lim _{n \rightarrow \infty} n\left(1-p_{00}^{(n+1)} / p_{00}^{(n)}\right)=\lim _{n \rightarrow \infty} n\left[1-B\left(A_{n}(0)\right)\right]>1$ and the second alternative occurs if this limit $<1$; the equality in the last expression follows from equation (2). The hypotheses and Taylor's theorem show that for $0 \leqq x<1, B(x)=1-\beta(1-x)+o(1-x)(x \uparrow 1)$ and since $A_{n}(0) \uparrow 1(n \rightarrow \infty)$ (Harris [3]) we have

$$
n\left[1-B\left(A_{n}(0)\right)\right]=\beta n\left(1-A_{n}(0)\right)+n o\left(1-A_{n}(0)\right) \quad(n \rightarrow \infty)
$$

Theorem A, with $x=0$, shows that as $n \rightarrow \infty$ the right hand side of expression (4) tends to $\sigma$. 
We shall now show that under all the conditions stated in the theorem, $0<\lim _{n \rightarrow \infty} n^{\sigma} p_{00}^{(n)}<\infty$. From equation (2) we have

$$
n^{\sigma} p_{00}^{(n)}=B(0) \prod_{m=1}^{n-1}\left(\frac{m+1}{m}\right)^{\sigma} B\left(A_{m}(0)\right)=B(0) \prod_{m=1}^{n-1} D_{m}
$$

where $D_{m}=(1+1 / m)^{\sigma} B\left(A_{m}(0)\right)$. A necessary and sufficient condition for the required limit to exist and be finite and positive is that $-\infty<\sum_{m=1}^{\infty}\left(D_{m}-1\right)<\infty$. Theorem A shows that $1-A_{m}(0)=1 /\left(1-h_{m}+m \gamma\right)$ where $h_{m}=o(m)(m \rightarrow \infty)$. Using this fact, a three term Taylor expansion of $B(x)$ to the left of $x=1$, and the fact that $(1+1 / m)^{\sigma}=1+\sigma / m+O\left(1 / m^{2}\right)$ enables us to write

$$
\begin{aligned}
D_{m}-1 & =\frac{\sigma}{m}-\frac{\beta}{m \gamma+1-h_{m}}+O\left(1 / m^{2}\right) \\
& =\frac{\sigma\left(1-h_{m}\right) / \gamma}{m^{2}+m\left(1-h_{m}\right) / \gamma}+O\left(1 / m^{2}\right)
\end{aligned}
$$

It is clear that $\sum_{m=1}^{\infty}\left(D_{m}-1\right)$ will converge to a finite limit if $\sum_{m=1}^{\infty} h_{m} / m^{2}$ does so. In fact Harris [3] shows that if $A^{\prime \prime \prime}(1-)<\infty$, that is $\delta \geqq 1,\left[h_{m}\right]=O(\log m)$ so in this case the series converges. We now consider the case $0<\delta<1$.

Using expression (3), the non-increasing nature of $\varepsilon(x)$ and the fact that $A_{n}(0) \uparrow 1$ $(n \rightarrow \infty)$, we obtain

$$
\frac{-\gamma^{2}}{1-a_{0}} \sum_{m=1}^{\infty} \frac{1}{m^{2}} \sum_{k=0}^{m-1}\left(1-A_{k}(0)\right) \leqq \sum_{m=1}^{\infty} \frac{h_{m}}{m^{2}} \leqq \sum_{m=1}^{\infty} \frac{1}{m^{2}} \sum_{k=0}^{m-1} \varepsilon\left(A_{k}(0)\right)
$$

For sufficiently large $n$ there exist positive constants $a, b$ such that $a / n<1-A_{n}(0)$ $<b / n$ and so we see that the terms of the series on the left of equation (5) are $O\left[(\log m) / m^{2}\right]$ for large $m$. Using the condition on $\varepsilon(x)$ given in the statement of the theorem and also that $0 \leqq \varepsilon(x) \leqq \gamma(0 \leqq x<1)$, it is not difficult to show that the terms on the right hand of equation (5) are $O\left(m^{-1-\delta}\right)$ for large $m$. The proof is now complete.

\section{REMARKS}

1. By an argument very similar to that used in the first part of the proof of lemma 8 of Kesten et al. (1966), it follows that the condition on $\varepsilon(x)$ given in theorem 1 above may be replaced by $\sum_{j=1}^{\infty} a_{j} j^{2} \log j<\infty$.

2. In obtaining the asymptotic form of $p_{00}^{(n)}$ we have not made use of irreducibility; we only require $A(0), \gamma>0$ and that $0<B(0)<1$, that is, there is positive probability of no immigrants in any generation.

3. By way of example, let $A(x)=1 /(2-x)$ and $B(x)=[1 /(2-x)]^{v}(v>0)$. Using the fractional linear generating function in Harris [3] p. 9, we see that $p_{00}^{(n)} \sim n^{-v}(n \rightarrow \infty)$. If instead we have a Poisson immigration component, that is $B(x)=e^{-\beta(1-x)}$, then $p_{00}^{(n)} \sim e^{-\eta \beta} n^{-\beta}$, where $\eta$ is Euler's constant. 
Since the Markov chain is transient when $\sigma>1$, the zero state is entered only finitely often with probability one, that is, with probability one, after a finite number of generations have passed the population size will always be positive. The situation when $\sigma \leqq 1$ is of course different, and the following theorem gives some information on this matter.

Theorem 2. Let $\alpha=1,0<B(0), \beta, \gamma<\infty, B(0)<1$ and either let $\varepsilon(x)=$ $O\left[(1-x)^{\delta}\right](\delta>0,0 \leqq x<1)$ or $\sum_{j=1}^{\infty} a_{j} j^{2} \log j<\infty$, so that $p_{00}^{(n)} \sim C n^{-\sigma}$ $(0<C<\infty)$ and let $\sigma \leqq 1$. Define the sequence $\left\{U_{n}\right\}(n=1,2, \cdots)$ by $U_{n}=$ $C \Gamma(1-\sigma) n^{1-\sigma}$ if $\sigma<1$ and $U_{n}=C \log n$ if $\sigma=1$. Finally let $V(\cdot)$ be the indicator function of the zero state, that is $V(j)=1$ if $j=0$, and $V(j)=0$ otherwise. Then if $0<\sigma \leqq 1$, we have

$$
\lim _{n \rightarrow \infty} \operatorname{Pr}\left\{\frac{1}{U_{n}} \sum_{m=0}^{n} V\left(X_{m}\right) \leqq x\right\}=G_{1-\sigma}(x)
$$

where $G_{\xi}(x)$ is the Mittag-Leffler distribution function given by

if $1>\xi \geqq 0$.

$$
G_{\xi}(x)=\frac{1}{\pi \xi} \int_{0}^{x} \sum_{j=0}^{\infty} \frac{(-)^{j-1}}{j !} \sin (\pi \xi j) \Gamma(\xi j+1) y^{j-1} d y
$$

Proof. Use of the inequality

$$
\int_{v}^{n+1} x^{-\sigma} d x<\sum_{m=v}^{n} m^{-\sigma}<\int_{v-1}^{n} x^{-\sigma} d x
$$

where $v, n$ are positive integers and $n>v$ shows that

$$
\sum_{m=0}^{n} p_{00}^{(m)} \sim\left\{\begin{array}{ll}
C n^{1-\sigma} /(1-\sigma) & \text { if } \sigma<1 \\
C \log n & \text { if } \sigma=1
\end{array} \quad(n \rightarrow \infty)\right.
$$

An Abelian theorem then shows that

$$
\sum_{n=0}^{\infty} p_{00}^{(n)} x^{n} \sim\left\{\begin{array}{ll}
C \Gamma(1-\sigma)(1-x)^{-1+\sigma} & \text { if } \sigma<1 \\
-C \log (1-x) & \text { if } \sigma=1
\end{array} \quad(x \uparrow 1)\right.
$$

The conditions of the occupation time theorem of Darling and Kac [1] are seen to be fulfilled and the theorem follows.

\section{A limit theorem}

It is easy to show from (1) that when $\alpha=1$,

$$
E\left(X_{n} \mid X_{0}=i\right)=n \beta+i
$$

and so it seems appropriate to investigate the limit in some sense of $X_{-} \ln$ as $n \rightarrow \infty$. is ... cinsiucs culuvergence in distrioution, the following theorem shows that we get a non-trivial result. 
THEOREM 3. Let $\alpha=1$ and $0<\gamma, \beta, \sigma<\infty$. Then the sequence $\left\{X_{n} / n\right\}(n=$ $1,2, \cdots)$ tends in distribution to the gamma variate having the density function

$$
f(t)=\frac{1}{\beta \Gamma(\sigma)}\left(\frac{t}{\beta}\right)^{\sigma-1} e^{-t / \beta} \quad(t>0)
$$

Proof. It follows from equation (1) that $(\theta>0)$,

$$
\psi_{i}^{(n)}(\theta)=E\left(e^{-\theta X_{n} / n} \mid X_{0}=i\right)=\left[A_{n}\left(e^{-\theta / n}\right)\right]^{i} \prod_{m=0}^{n-1} B\left[A_{m}\left(e^{-\theta / n}\right)\right]
$$

Since $A_{n}(x) \uparrow 1(n \rightarrow \infty)$, we see that the first term on the right tends to unity. Writing $b_{m n}(\theta)=B\left[A_{m}\left(e^{-\theta / n}\right)\right]$, and using $\log (1-x) \geqq-x-x^{2} /(1-x)(0 \leqq x$ $<1$ ), we have

$$
\begin{aligned}
\phi^{(n)}(\theta)=\log \psi_{0}^{(n)}(\theta) & =\sum_{m=0}^{n-1} \log \left[1-\left(1-b_{m n}(\theta)\right)\right] \\
& =-\sum_{m=0}^{n-1}\left(1-b_{m n}(\theta)\right)+R_{1}^{(n)}(\theta)
\end{aligned}
$$

where

$$
\begin{aligned}
0 & \geqq R_{1}^{(n)}(\theta) \geqq-\sum_{m=0}^{n-1}\left[1-b_{m n}(\theta)\right]^{2} / b_{m n}(\theta) \\
& \geqq-\left[\left(1-b_{0 n}(\theta)\right) / b_{0 n}(\theta)\right] \sum_{m=0}^{n-1}\left(1-b_{m n}(\theta)\right)
\end{aligned}
$$

since $b_{m n}(\theta)$ is non-decreasing in $m$ for fixed $n$ and $\theta$. Since $1-b_{0 n}(\theta) \rightarrow 0(n \rightarrow \infty)$ it is clear that $R_{1}^{(n)}(\theta) \rightarrow 0$ if the first expression on the right hand side of equation (6) has a finite limit as $n \rightarrow \infty$.

For $0 \leqq x<1$, we have $1-B(x)=\beta(1-x)-(1-x) f(x)$ where $0 \leqq f(x)=$ $(1-x) B^{\prime \prime}(\xi) / 2$ and $x<\xi<1$ and $f(x)=o(1)$ as $x \uparrow 1$. Then letting $a_{m n}(\theta)=$ $A_{m}\left(e^{-\theta / n}\right)$, equation (6) becomes

where

$$
\phi^{(n)}(\theta)=-\beta \sum_{m=0}^{n-1}\left(1-a_{m n}(\theta)\right)+R_{1}^{(n)}(\theta)+R_{2}^{(n)}(\theta)
$$

$$
\begin{aligned}
0 \leqq R_{2}^{(n)}(\theta) & =\sum_{m=0}^{n-1}\left(1-a_{m n}(\theta)\right) f\left[a_{m n}(\theta)\right] \\
& \leqq\left(1-e^{-\theta / n}\right) \sum_{m=0}^{n-1} f\left(a_{m 1}(\theta)\right)
\end{aligned}
$$

since $f(x)$ is non-increasing. The last expression approaches zero $(n \rightarrow \infty)$ since $1-e^{-\theta / n} \sim \theta / n$ and $f\left[a_{n 1}(\theta)\right]=o(1)(n \rightarrow \infty)$.

Theorem A shows that we can write

$$
1-A_{m}(x)=\frac{1-x}{1+\gamma m(1-x)}\left[1+g_{m}(x)\right]
$$


where $g_{m}(x) \rightarrow 0$ uniformly in $0 \leqq x<1(m \rightarrow \infty)$ and $g_{m}(1)=0$. Thus we have $\phi^{(n)}(\theta)=-\beta \sum_{m=0}^{n-1} \frac{1-e^{-\theta / n}}{1+\gamma m\left(1-e^{-\theta / n}\right)}+R_{1}^{(n)}(\theta)+R_{3}^{(n)}(\theta)+o(1) \quad(n \rightarrow \infty)$

where

$$
R_{3}^{(n)}(\theta)=-\beta \sum_{m=0}^{n} \frac{1-e^{-\theta / n}}{1+\gamma m\left(1-e^{-\theta / n}\right)} g_{m}\left(e^{-\theta / n}\right)
$$

It follows from the uniform convergence of the $g_{m}(\cdot)$ that there exists $M(\varepsilon)$ such that $\left|g_{m}\left(e^{-\theta / n}\right)\right|<\varepsilon(n=1,2, \cdots)$ if $m>M(\varepsilon)$. Breaking the summation in expression (7) into the form $\sum_{m=0}^{M(\varepsilon)}+\sum_{m=M(\varepsilon)+1}^{n}$ and using the fact that

$$
\left[1-e^{-\theta / n}\right] /\left[1+\gamma m\left(1-e^{-\theta / n}\right)\right] \leqq 1-e^{-\theta / n} \sim \theta / n \quad(n \rightarrow \infty)
$$

shows that $R_{3}^{(n)}(\theta)=o(1)(n \rightarrow \infty)$.

It is easily seen that

$$
0 \leqq \frac{\theta / n}{1+\gamma m \theta / n}-\frac{1-e^{-\theta / n}}{1+\gamma m\left(1-e^{-\theta / n}\right)} \leqq \frac{\theta^{2}}{2 n^{2}}
$$

so that finally we have

$$
\phi^{(n)}(\theta)=-\beta \sum_{m=0}^{n-1} \frac{\theta / n}{1+\gamma m \theta / n}+R_{1}^{(n)}(\theta)+o(1) \quad(n \rightarrow \infty)
$$

The sum in this expression can be recognized as an upper Darboux sum of the Riemann integral

$$
\theta \int_{0}^{1}(1+\gamma \theta x)^{-1} d x
$$

so that we obtain

$$
\phi^{(n)}(\theta)=-\sigma \log (1+\gamma \theta)+o(1) \quad(n \rightarrow \infty)
$$

and thus $\lim _{n \rightarrow \infty} \psi_{i}^{(n)}(\theta)=(1+\gamma \theta)^{-\sigma}$ which is the Laplace transform of the density function given in the statement of the theorem. The convergence in distribution assertion follows from the continuity theorem for Laplace-Stieltjes transforms.

It is clear that the theorem is true under the conditions given in remark 2 following theorem 1 .

Added in proof. Since submitting this paper, the author has learned that theorem 3 was obtained independently by E. Seneta in J. Roy. Stat. Soc. 32 B (1970), 149-52.

\section{Acknowledgement}

This work was carried out during the tenure of a Department of Supply Post-graduate Studentship. 


\section{References}

[1] D. A. Darling, and M. Kac, On occupation times for Markov processes. Trans. Amer. Math. Soc. 84 (1957), 444-458.

[2] W. L. Ferrar, A Textbook of Convergence (O.U.P. Oxford, 1937).

[3] T. E. Harris, The Theory of Branching Processes. (Springer-Verlag, Berlin, 1963).

[4] C. R. Heathcote, A branching process allowing immigration. J. Roy. Stat. Soc. Ser. B. 27 (1965), 138-43.

[5] C. R. Heathcote, Corrections and comments on the paper 'A branching process allowing immigration'. J. Roy. Stat. Soc. Ser. B, 28 (1966), 213-17.

[6] H. Kesten, P. Ney, and F. Spitzer, The Galton-Watson process with mean one and finite variance. Teor. Veroyatnost. i Primenen., 11 (1966) 579-611.

[7] E. Seneta, The stationary distribution of a branching process allowing immigration: a remark on the critical case. J. Roy. Stat. Soc., Ser B, 30 (1968) 176-9.

Department of Mathematics

Monash University

Melbourne, Australia 